\title{
Accuracy of a Factory-Calibrated, Real-Time Continuous Glucose Monitoring System During 10 Days of Use in Youth and Adults with Diabetes
}

\author{
R. Paul Wadwa, MD, Lori M. Laffel, MD, MPH, Viral N. Shah, MD, and Satish K. Garg, MD ${ }^{1}$
}

\begin{abstract}
Background: Frequent use of continuous glucose monitoring (CGM) systems is associated with improved glycemic outcomes in persons with diabetes, but the need for calibrations and sensor insertions are often barriers to adoption. In this study, we evaluated the performance of G6, a sixth-generation, factory-calibrated CGM system specified for 10-day wear.

Methods: The study enrolled participants of ages 6 years and up with type 1 diabetes or insulin-treated type 2 diabetes at 11 sites in the United States. Participation involved one sensor wear period of up to 10 days. Adults wore the system on the abdomen; youth of ages 6-17 years could choose to wear it on the abdomen or upper buttocks. Clinic sessions for frequent comparison with reference blood glucose measurements took place on days $1,4-5,7$, and/or 10. Participants of ages 13 years and up underwent purposeful supervised glucose manipulation during in-clinic sessions. During the study, participants calibrated the systems once daily. However, analysis was performed on glucose values that were derived from reprocessed raw sensor data, independently of self-monitored blood glucose values used for calibration. Reprocessing used assigned sensor codes and a factory-calibration algorithm. Performance evaluation included the proportion of CGM values that were within $\pm 20 \%$ of reference glucose values $>100 \mathrm{mg} / \mathrm{dL}$ or within $\pm 20 \mathrm{mg} / \mathrm{dL}$ of reference glucose values $\leq 100 \mathrm{mg} / \mathrm{dL}$ $(\% 20 / 20)$, the analogous \%15/15, and the mean absolute relative difference (MARD, expressed as a percentage) between temporally matched CGM and reference values.

Results: Data from 262 study participants (21,569 matched CGM reference pairs) were analyzed. The overall $\% 15 / 15, \% 20 / 20$, and MARD were $82.4 \%, 92.3 \%$, and $10.0 \%$, respectively. Matched pairs from 134 adults and 128 youth of ages 6-17 years were similar with respect to \%20/20 (92.4\% and 91.9\%) and MARD (9.9\% and $10.1 \%$ ). Overall $\% 20 / 20$ values on days 1 and 10 of sensor wear were $88.6 \%$ and $90.6 \%$, respectively. The system's "Urgent Low Soon" (predictive of hypoglycemia within $20 \mathrm{~min}$ ) hypoglycemia alert was correctly provided $84 \%$ of the time within 30 min before impending biochemical hypoglycemia $(<70 \mathrm{mg} / \mathrm{dL})$. The 10 -day sensor survival rate was $87 \%$.
\end{abstract}

Conclusion: The new factory-calibrated G6 real-time CGM system provides accurate readings for 10 days and removes several clinical barriers to broader CGM adoption.

Keywords: Glucose sensor performance, Clinical accuracy, MARD, Continuous glucose monitoring, Advanced algorithm, Factory-calibrated.

\section{Introduction}

I MPROVEMENTS IN CONTINUOUS glucose monitoring (CGM) systems have contributed to their increased adoption for people with type 1 diabetes (T1D) ${ }^{1}$ and for some people with insulin-treated type 2 diabetes (T2D). The systems are now frequently used to guide diabetes management decisions related to insulin dosing, carbohydrate intake, and exercise. However, the requirement for twice-daily calibrations with self-monitored blood glucose (SMBG) values and the need to

\footnotetext{
${ }^{\mathrm{I}}$ Barbara Davis Center for Diabetes, University of Colorado Denver, Aurora, Colorado.

${ }^{2}$ Joslin Diabetes Center, Harvard Medical School, Boston, Massachusetts.
} 
replace the sensor every 7 days contribute to the cost and inconvenience of currently marketed real-time (rt)CGM systems.

Until recently, all CGM systems required calibration during use. Sensor technology has now advanced such that sensor-specific calibrations can be conducted during the manufacturing process (i.e., factory calibration). ${ }^{2,3}$ A factorycalibrated rtCGM system-G6-was developed and recently approved by the FDA. 4

The system incorporates several advancements including a permselective membrane to limit the interference effect of acetaminophen and an automated sensor applicator ${ }^{5}$ intended to simplify the insertion and result in more consistent sensor deployment. Three related studies were performed to assess G6 system performance. A small study ${ }^{6}$ established the system's resistance to acetaminophen interference. A second study, in which participants used an automated sensor applicator to insert the G6 sensor, is the subject of a separate article and reflects the commercially marketed system.

The study described here evaluated sensor accuracy when deployed with a manual applicator, similar to that currently used with G4 and G5 sensors, and was performed to evaluate sensor performance independently of applicator device. Participants of ages 13 years and up underwent closely monitored glucose manipulations to evaluate sensor performance in low and high glucose ranges.

\section{Methods}

\section{Study design}

The G6 rtCGM system (Dexcom, Inc., San Diego, CA) was evaluated in a prospective multicenter study enrolling 139 adults of ages 18 years and up and 151 youth of ages 6-17 years with T1D or insulin-requiring T2D at 11 U.S. sites. Inclusion criteria comprised use of intensive insulin therapy (either multiple daily injections or pump therapy) with known insulin dosing parameters for at least 3 months before study enrollment; willingness to perform at least seven SMBG tests per day during home use with the study-assigned blood glucose meter (Contour NEXT ONE Blood Glucose Meter, Ascensia Diabetes Care, Parsippany, NJ) for oncedaily calibration, diabetes management, alert verification, and comparative purposes; and, for participants of 13 years and older, willingness to undergo deliberate insulin and glucose challenges to induce supervised mild-to-moderate hypoand hyperglycemia. Exclusion criteria comprised the presence of extensive skin abnormalities at the sensor insertion site, pregnancy, hematocrit values outside the normal range, or any condition that could be negatively impacted by glucose manipulation.

\section{Device description}

Some of the improvements to the commercial G5 Mobile CGM system that are incorporated into the G6 CGM system include factory calibration (although users can still enter calibrations if they wish to more closely align CGM data with their home glucose meter), acetaminophen-blocking sensor membrane, smaller and thinner transmitter, 10-day sensor wear, and predictive low glucose alert ("Urgent Low Soon" alert). The "Urgent Low Soon" alert is triggered when the glucose concentration is predicted to reach $55 \mathrm{mg} / \mathrm{dL}$ within $20 \mathrm{~min}$.

\section{Study procedures and data collection}

Participants received training on the system using prepared training materials; all sensor insertions were performed at the clinic by participants and/or guardians. Participants or their guardians deployed the sensors using a manual sensor applicator (similar to that used with Dexcom G4 and G5 sensors). All participants used the G6 system for one 10-day wear period (up to $240 \mathrm{~h}$ ). Adult participants wore the sensor on the abdomen. Youth of ages 6-17 years could choose to wear the sensor on the abdomen or upper buttocks. Sensors that failed within the first $12 \mathrm{~h}$ after insertion were replaced.

Participants were scheduled for one or three in-clinic sessions of varying duration on days 1, 4-5, 7, and/or 10 of system use based on their age group. Adults returned for up to three 12-hour sessions. Participants of ages 13-17 years were scheduled for one 12-hour clinic session. Participants of ages 6-12 years were scheduled for one 6-hour clinic session. All sessions included comparison of CGM readings with venous glucose concentrations using a laboratory reference method (Yellow Springs Instrument (YSI); YSI, Inc., Yellow Springs, $\mathrm{OH})$. Participants had venous blood drawn once every $15 \pm 5 \mathrm{~min}$ for the duration of each clinic session for measurement on the YSI. A heating pad was applied around the intravenous site to "arterialize" the venous sample, allowing a closer match between venous and capillary glucose concentrations. The CGM

Table 1. Demographics and Baseline Characteristics of Analysis Population $(N=262)$

\begin{tabular}{lc}
\hline Characteristic & $\mathrm{n}(\%)$ \\
\hline Age (years) & \\
Children (6-12) & $60(23)$ \\
Adolescents (13-17) & $68(26)$ \\
Adults $(18-59)$ & $111(42)$ \\
Seniors (60+) & $23(9)$ \\
Mean \pm SD & $28.0 \pm 18.3$ \\
Gender & \\
Male & $124(47)$ \\
Female & $138(53)$ \\
Ethnicity & \\
Hispanic or Latino & $25(10)$ \\
Not Hispanic or Latino & $237(90)$ \\
Type of diabetes & \\
Type 1 & $260(99.2)$ \\
Type 2 & $2(0.8)$ \\
Diabetes duration (years) & \\
$0-4$ & $66(25)$ \\
$5-9$ & $64(24)$ \\
$10-14$ & $35(13)$ \\
15-19 & $28(11)$ \\
20+ & $69(26)$ \\
Mean \pm SD & $15.1 \pm 13.8$ \\
HbA1c (\%) & \\
$<7.0$ & $52(20)$ \\
$7.0-<7.5$ & $50(19)$ \\
$7.5-<8.0$ & $45(17)$ \\
$8.0-<8.5$ & $37(14)$ \\
$\geq 8.5$ & $78(30)$ \\
Mean \pm SD & $8.0 \pm 1.3$ \\
\hline
\end{tabular}

Data shown are presented as $n(\%)$ or mean \pm SD unless otherwise noted. 
Table 2. Overall Continuous Glucose Monitoring Performance by Subject Age Group

\begin{tabular}{lcccccc}
\hline & \multirow{2}{*}{$\begin{array}{c}\text { Subjects } \\
\text { Population }\end{array}$} & $\begin{array}{c}\text { Matched } \\
\text { pairs (n) }\end{array}$ & $\%(95 \%$ LB) & $\%(95 \%$ LB) & $\%(95 \%$ LB) & $\%(95 \%$ UB) \\
\hline Overall & 262 & 21,569 & $82.4(80.5)$ & $92.3(91.2)$ & $98.7(98.3)$ & $10.0(10.4)$ \\
Adults (ages 18+) & 134 & 17,184 & $82.6(80.4)$ & $92.4(91.2)$ & $98.8(98.5)$ & $9.9(10.4)$ \\
Youth (ages 6-17) & 128 & 4385 & $81.6(78.1)$ & $91.9(89.4)$ & $97.9(96.9)$ & $10.1(11.0)$ \\
\hline
\end{tabular}

95\% LB, lower bound of the one-sided 95\% confidence interval; $95 \%$ UB, upper bound of the one-sided $95 \%$ confidence interval; MARD, mean absolute relative difference.

data were masked to participants and clinic staff for the duration of the clinic session. During the clinic session(s), subjects had at least two fingerstick blood glucose tests taken hourly by the subject or study staff at the investigator's (or qualified staff) discretion to confirm, detect, or help manage hypoglycemia and hyperglycemia.

To ensure that CGM data adequately covered the system's entire reportable range $(40-400 \mathrm{mg} / \mathrm{dL})$ and each glucose range was adequately represented, glucose levels were manipulated during the clinic sessions for 202 participants of ages 13 years and older. Under close observation by the study investigator staff, per protocol, participant glucose levels were deliberately manipulated to induce high or low blood glucose levels through carbohydrate consumption and insulin timing. Target glucose ranges included Level 2 hypoglycemia $(<54 \mathrm{mg} / \mathrm{dL})$, Level 1 hypoglycemia (54-69 mg/dL), euglycemia $(70-180 \mathrm{mg} / \mathrm{dL})$, Level 1 hyperglycemia $(181-250 \mathrm{mg} / \mathrm{dL})$, and Level 2 hyperglycemia range $(>250 \mathrm{mg} / \mathrm{dL})^{8}{ }^{8}$

Safety was monitored using SMBG testing. The glucose manipulation protocol was reviewed and approved through an Investigation Device Exemption application (G160069) before study initiation. The study was reviewed by the FDA through the investigational device exemption process and registered at CinicalTrials.gov (NCT02880267). The local IRB approved all study procedures before study initiation and all participants and/or legal guardian provided written informed consent/assent before any study procedures.

\section{Methods of data analysis}

Analysis was performed on glucose values that were derived from reprocessed raw sensor data, independently of SMBG values used for calibration. Reprocessing used assigned sensor codes and a factory-calibration algorithm. The sensor code accounts for intersensor variation and is developed for each sensor individually during manufacturing based on the unique performance characteristics of each sensor. Only reprocessed factory-calibration data are reported in this study.
Each YSI value was paired with the CGM value that immediately followed (within $5 \mathrm{~min}$ ); these matched pairs formed the analysis data set. CGM-YSI matched pairs within the CGM reportable range were evaluated. The CGM-YSI matched pair data available from clinic-session participants without major protocol deviations were included in the analysis.

Accuracy metrics included the proportion of the CGM system values that were within $\pm 20 \%$ of paired YSI values $>100 \mathrm{mg} / \mathrm{dL}$ or $\pm 20 \mathrm{mg} / \mathrm{dL}$ of YSI values $\leq 100 \mathrm{mg} / \mathrm{dL}$ (hereafter referred to as \%20/20), as well the analogous $\% 15 / 15$ and $\% 30 / 30$. The bootstrapped one-sided $95 \%$ lower bound (95\% LB) was calculated for $\% 15 / 15, \% 20 / 20$, and $\% 30 / 30$ accuracies. The mean absolute relative difference (MARD) was determined as the average of the relative difference between paired CGM and YSI values; MARD and the corresponding bootstrapped one-sided $95 \%$ upper bound $(95 \%$ UB) were calculated for all CGM-YSI pairs; MARD and standard error (SE) were calculated when the CGM value was $\geq 70 \mathrm{mg} / \mathrm{dL}$. Mean absolute difference (MAD) was determined as the average of the absolute difference between paired CGM and YSI values when CGM values were $<70 \mathrm{mg}$ / $\mathrm{dL}$; the corresponding SE was calculated.

The ability of the G6 system to alarm appropriately when alerts were set at different thresholds was assessed by comparing CGM readings with YSI measurements at low $(55,60$, $70,80$, and $90 \mathrm{mg} / \mathrm{dL})$ and high $(120,140,180,200,220,240$, and $300 \mathrm{mg} / \mathrm{dL}$ ) glucose threshold levels and determining whether an alert would have sounded. For all thresholds, two metrics were assessed: alert rate, which evaluates whether YSI is in agreement with the device when the device alerts, and detection rate, which evaluates whether the device alerts when YSI is within a certain threshold. At low glucose thresholds, true alert rate was defined as the percentage of times in which the YSI blood glucose level was at or below the threshold alert setting $15 \mathrm{~min}$ before or after the alert would have been triggered. The false alert rate was defined as $(1-$ true alert rate).

The correct detection rate at low glucose thresholds was defined as the frequency of CGM alerts occurring within

Table 3. Continuous Glucose Monitoring Performance Across Glucose Ranges $(N=262)$

\begin{tabular}{|c|c|c|c|c|c|c|}
\hline $\begin{array}{l}\text { CGM level } \\
(m g / d L)\end{array}$ & $\begin{array}{l}\text { Matched } \\
\text { pairs (n) }\end{array}$ & $\begin{array}{c}\% 15 / 15 \\
(\%)\end{array}$ & $\begin{array}{c}\% 20 / 20 \\
(\%)\end{array}$ & $\begin{array}{c}\% 30 / 30 \\
(\%)\end{array}$ & $\begin{array}{c}M A D \\
m g / d L(S E)\end{array}$ & $\begin{array}{c}M A R D \% \\
\quad(S E)\end{array}$ \\
\hline$<54$ & 447 & 78.7 & 85.0 & 93.5 & $10.9(0.7)$ & N.A. \\
\hline $54-69$ & 1640 & 89.5 & 94.4 & 97.7 & $7.8(0.2)$ & N.A. \\
\hline $70-180$ & 9879 & 78.2 & 89.9 & 98.2 & N.A. & $11.1(0.1)$ \\
\hline $181-250$ & 4902 & 80.8 & 92.4 & 99.3 & N.A. & $9.2(0.1)$ \\
\hline$>250$ & 4701 & 90.7 & 97.4 & 99.9 & N.A. & $7.2(0.1)$ \\
\hline
\end{tabular}

CGM, continuous glucose monitoring; MAD, mean absolute difference; N.A., not applicable; MARD, mean absolute relative difference; SE, standard error. 
Table 4. Continuous Glucose Monitoring Performance Across Days of Sensor Wear $(N=262)$

\begin{tabular}{lcccrr}
\hline Clinic session day & Matched pairs (n) & $\% 15 / 15(\%)$ & $\% 20 / 20(\%)$ & $\% 30 / 30(\%)$ & $M A R D \%(S E)$ \\
\hline Day 1 & 3720 & 75.4 & 88.6 & 98.1 & $11.5(0.2)$ \\
Day 2 & 3760 & 81.6 & 90.9 & 98.6 & $10.5(0.1)$ \\
Days 4-5 & 6852 & 85.2 & 95.0 & 99.3 & $9.2(0.1)$ \\
Day 7 & 3734 & 86.5 & 94.2 & 99.1 & $8.9(0.1)$ \\
Day 10 & 3503 & 80.7 & 90.6 & 97.5 & $10.6(0.2)$ \\
\hline
\end{tabular}

15 min of a YSI blood glucose below the threshold setting. The missed detection rate at low glucose thresholds was defined as (1 - correct detection rate).

An additional analysis was performed to determine how often biochemical hypoglycemia (YSI glucose $<70 \mathrm{mg} / \mathrm{dL}$ ) was detected when low glucose thresholds were set at 55 or $60 \mathrm{mg} / \mathrm{dL}$.

At high glucose thresholds, the true alert rate was defined as the percentage of occasions in which the YSI blood glucose level was at or above the threshold alert setting $15 \mathrm{~min}$ before or after the alert would have been triggered. The false alert rate at high glucose thresholds was defined as ( 1 - true alert rate).

The correct detection rate at low glucose thresholds was defined as the frequency of CGM alerts occurring within 15 min of a YSI blood glucose above the threshold setting. The missed detection rate at high glucose thresholds was defined as ( 1 - correct detection rate).

For the analysis of "Urgent Low Soon" alert, which is triggered when the glucose concentration is predicted to reach $55 \mathrm{mg} / \mathrm{dL}$ within $20 \mathrm{~min}$, actualized hypoglycemia (either YSI $<55$ or $<70 \mathrm{mg} / \mathrm{dL}$ ) was evaluated within $30 \mathrm{~min}$ of an Urgent Low Soon alert to accommodate the YSI sampling schedule. Individuals with a CGM glucose measurement $>70 \mathrm{mg} / \mathrm{dL}$ who had not eaten 10 minutes before to 20 minutes after the activation of the Urgent Low Soon alert were included in this alert performance analysis.

Trend accuracy assessed the concurrence between rates of glucose change measured by YSI and CGM. Rate of change (RoC) was calculated by interpolating per minute glucose concentrations for YSI and CGM data separately. Six RoC categories $(<-2,-2$ to $<-1,-1$ to $<0,0$ to $<1,1$ to 2 , and $>2 \mathrm{mg}$ / $(\mathrm{dL} \cdot \mathrm{min}))$ were established. The percentage of time that the CGM-measured RoC matched the YSI-measured RoC within one category on either side was assessed. In addition, root-meansquare (RMS) error was calculated for the RoC measured by CGM compared with the RoC measured by YSI. Point accuracy was also determined at different CGM rates of glucose change.

To estimate time lag, CGM measurements were interpolated to provide values at 1-minute intervals. Each of the measured and interpolated CGM values was associated with a delay time between it and the most recently preceding YSI value. The absolute relative difference (ARD) between each CGM value and its associated YSI value was calculated. The delay time associated with the CGM value having the lowest ARD was taken as the time lag for that subject. Summary statistics for subjects' delay times are presented. All analyses were performed using SAS ${ }^{\circledR}$ software, version 9.3 (SAS Institute, Inc., Cary, NC).

\section{Results \\ Study population}

Two hundred and ninety participants with T1D or insulintreated T2D were enrolled. Twenty-eight participants were excluded from the accuracy assessment: 2 participants swapped receivers, 4 participants had a transmitter download failure, 19 had no YSI data, and 3 had no matching sensor data to YSI data. Thus, 262 participants contributed CGMYSI matched pairs for the accuracy assessment. Demographics and baseline characteristics of the analysis cohort are given in Table 1.

\section{CGM performance with reprocessed factory calibration}

Overall MARD. A total of 21,760 CGM-YSI matched pair measurements were available. Among these, 191 matched pairs $(0.9 \%)$ had CGM readings out of the reportable range; 137 had "LOW" CGM readings ( $<40 \mathrm{mg} / \mathrm{dL})$, of which $58.3 \%$ had YSI readings $\leq 60 \mathrm{mg} / \mathrm{dL}$; and 54 had " $\mathrm{HIGH}$ " CGM readings ( $>400 \mathrm{mg} / \mathrm{dL}$ ), of which $94.4 \%$ had YSI readings $>350 \mathrm{mg} / \mathrm{dL}$. Because distinct CGM measurements are not provided $<40$ or $>400 \mathrm{mg} / \mathrm{dL}$, these matched pairs could not be used to calculate point or percentage accuracy. Therefore, 21,569 CGM-YSI matched pair measurements were used for analysis. The MARD (95\% UB) between CGM and YSI values was $10.0 \%$ (10.4) for all participants, 9.9\% (10.4) for adults of ages 18+ years, and $10.1 \%$ (11.0) for youth of ages 6-17 years (Table 2).

Overall percentage accuracy, percentage accuracy across glucose ranges, and percentage and point accuracy across days of wear. The G6 system showed a \%20/20 accuracy (95\% LB) of $92.3 \%$ (91.2) for all participants; $92.4 \%(91.2)$ for adults, and $91.9 \%$ (89.4) for youth (Table 2). The \%15/15 accuracy was consistently $\geq 81 \%$ across age groups, whereas the $\% 30 / 30$ accuracy was not $<97.9 \%$ for any age group (Table 2).

Purposeful glucose manipulation allowed assessment of accuracy across hypo- and hyperglycemic glucose ranges. From 21,569 evaluated matched pairs, $9.7 \%$ (2087) were measured in the Level 1 or Level 2 hypoglycemic range, whereas $21.8 \%$ (4701) were measured in the Level 2 hyperglycemic range. The

Table 5. Continuous Glucose Monitoring Performance at Various Rates of Change $(N=262)$

\begin{tabular}{lccr}
\hline $\begin{array}{l}\text { Matched } \\
\text { pairs }(\mathrm{n})\end{array}$ & $\begin{array}{c}\text { CGM rate } \\
\text { of change } \\
(\mathrm{mg} /(\mathrm{dL} \cdot \text { min }))\end{array}$ & $\begin{array}{c}\% 20 / 20 \\
(\%)\end{array}$ & $\begin{array}{c}\text { MARD } \\
\%(S E)\end{array}$ \\
\hline 634 & $<-2$ & 89.7 & $10.1(0.4)$ \\
2489 & {$[-2,-1)$} & 93.0 & $9.6(0.2)$ \\
8427 & {$[-1,0)$} & 93.9 & $10.0(0.1)$ \\
5741 & {$[0,1)$} & 92.7 & $9.9(0.1)$ \\
2049 & {$[1,2]$} & 89.6 & $9.9(0.2)$ \\
1624 & $>2$ & 87.1 & $10.8(0.2)$ \\
\hline
\end{tabular}


Table 6. Trend Accuracy $(N=262)$

\begin{tabular}{lccccccc}
\hline \multirow{2}{*}{$\begin{array}{l}\text { Matched } \\
\text { pairs }(\mathrm{n})\end{array}$} & $\begin{array}{c}\text { CGM rate } \\
\text { of change } \\
(\mathrm{mg} /(\text { dL·min }))\end{array}$ & $<-2$ & {$[-2,-1)$} & {$[-1,0)$} & {$[0,1)$} & {$[1,2]$} & $>2$ \\
\cline { 3 - 7 } & $<-2$ & $52.1 \%$ & $35.5 \%$ & $10.4 \%$ & $1.7 \%$ & $0.0 \%$ & $0.3 \%$ \\
634 & {$[-2,-1)$} & $7.6 \%$ & $56.2 \%$ & $32.7 \%$ & $3.1 \%$ & $0.4 \%$ & $0.1 \%$ \\
2489 & {$[-1,0)$} & $0.5 \%$ & $9.9 \%$ & $75.7 \%$ & $13.1 \%$ & $0.7 \%$ & $0.1 \%$ \\
8427 & {$[0,1)$} & $0.1 \%$ & $1.1 \%$ & $26.3 \%$ & $60.0 \%$ & $10.9 \%$ & $1.6 \%$ \\
5741 & {$[1,2]$} & $0.0 \%$ & $0.4 \%$ & $3.6 \%$ & $28.3 \%$ & $51.8 \%$ & $15.9 \%$ \\
2049 & $>2$ & $0.1 \%$ & $0.2 \%$ & $0.9 \%$ & $5.9 \%$ & $21.9 \%$ & $71.0 \%$ \\
1624 & & & & & & &
\end{tabular}

YSI, yellow springs instrument.

$\% 20 / 20$ of the G6 system was $85.0 \%$ in the Level 2 hypoglycemic range, $94.4 \%$ in the Level 1 hypoglycemic range, $89.9 \%$ in the euglycemic range, $92.4 \%$ in the Level 1 hyperglycemic range, and $97.4 \%$ in the Level 2 hyperglycemia range (Table 3). The corresponding \%15/15 and \%30/30 accuracies across glucose ranges, as well as MAD in the hypoglycemic range and MARD in the euglycemic and hyperglycemic ranges, are given in Table 3 .

The G6 system's accuracy across days of sensor wear was evaluated using percentage and point accuracy (Table 4). The $\% 20 / 20$ and $\% 15 / 15$ on the first day of wear were $88.6 \%$ and $75.4 \%$, respectively. The $\% 20 / 20$ and $\% 15 / 15$ on day 10 of sensor wear were $90.6 \%$ and $80.7 \%$, respectively (Table 4). MARD values (SE) on days 1 and 10 were $11.5 \%(0.2)$ and $10.6 \%(0.2)$, respectively (Table 4).

Percentage and point accuracy at various ROCs. The system's performance at different glucose concentration ROCs was assessed (Table 5). During rapid ROCs $(<-2$ or $>2 \mathrm{mg} /(\mathrm{dL} \cdot \mathrm{min}))$, the $\% 20 / 20$ values were $89.7 \%$ and $87.1 \%$, respectively; corresponding MARD values (SE) were $10.1 \%$ (0.4) and $10.8 \%$ (0.2). For ROCs $\geq-2$ and $\leq 2 \mathrm{mg} /(\mathrm{dL} \cdot \mathrm{min})$, the $\% 20 / 20$ values were consistently $\geq 89.6 \%$ and the MARD values were consistently $\leq 10 \%$.

Trend accuracy. Trend accuracy was also assessed at different rates of glucose concentration change. When the CGM values were rising rapidly $(>2 \mathrm{mg} /(\mathrm{dL} \cdot \mathrm{min}))$, the reference glucose was rising $98.8 \%$ of the time (Table 6$)$. When the CGM values were falling rapidly $(<-2 \mathrm{mg} /(\mathrm{dL} \cdot \mathrm{min}))$, the reference glucose was falling $98.0 \%$ of the time (Table 6). Conversely, when CGM values were changing by no more than $1 \mathrm{mg} /(\mathrm{dL} \cdot \mathrm{min})$, reference glucose values were changing rapidly $(>2 \mathrm{mg} /(\mathrm{dL} \cdot \mathrm{min})$ or $<-2 \mathrm{mg} /(\mathrm{dL} \cdot \mathrm{min}))$ only $2.3 \%$ of the time. RMS error for CGM-measured RoC compared with YSI-measured RoC was $0.6 \mathrm{mg} / \mathrm{dL}$ per minute. The concordance rate between CGM-measured RoC and YSI-measured RoC within one category was $97.2 \%$.

Threshold alert and Urgent Low Soon alert performance. When the hypoglycemia threshold alert was set to $70 \mathrm{mg} / \mathrm{dL}$, the true alert rate was $84.4 \%$ and the correct detection rate was $85.0 \%$; performance of the low glucose alert at multiple thresholds is presented in Table 7 . If an alert was provided when CGM value fell below the glucose thresholds of 55 or $60 \mathrm{mg} / \mathrm{dL}, 85 \%$ and $87 \%$ of the time YSI glucose was $<70 \mathrm{mg} / \mathrm{dL}$. When the hyperglycemia threshold alert was set at $240 \mathrm{mg} / \mathrm{dL}$, the true alert rate was $93.2 \%$ and the correct detection rate was $90.0 \%$; performance of the high glucose alert at multiple thresholds is presented in Table 8.

Of the 44 "Urgent Low Soon" alert activations, 37 (84\%) were followed by an event defined at $<70 \mathrm{mg} / \mathrm{dL}$ within 30 min of which $21(48 \%)$ were followed by a hypoglycemic event defined at $<55 \mathrm{mg} / \mathrm{dL}$ within $30 \mathrm{~min}$.

Sensor survival. Eighty-seven percent of the sensors lasted through the end of the entire 10-day wear period. Sensor survival probability and its $95 \%$ confidence interval as a function of sensor day are shown in Figure 1. Among the 285 sensors evaluated, 7 (2.5\%) failed due to an adhesive failure and $24(8.4 \%)$ failed due to early sensor shut-off.

Time lag. Sensors worn by 262 individuals provided data for time lag analysis. The mean (SD) time lag was 4.5 (3.3) minutes.

Table 7. Hypoglycemic Alerts and Detections $(N=262)$

\begin{tabular}{|c|c|c|c|c|c|c|}
\hline \multirow[b]{2}{*}{$\begin{array}{l}\text { Hypoglycemic } \\
\text { alert level } \\
(\mathrm{mg} / \mathrm{dL})\end{array}$} & \multicolumn{3}{|c|}{ Alerts } & \multicolumn{3}{|c|}{ Detections } \\
\hline & $\begin{array}{l}\text { No. of } \\
\text { alerts (n) }\end{array}$ & $\begin{array}{c}\text { True alert } \\
\text { rate }(\%)\end{array}$ & $\begin{array}{c}\text { False alert } \\
\text { rate }(\%)\end{array}$ & $\begin{array}{c}\text { No. of } \\
\text { events (n) }\end{array}$ & $\begin{array}{l}\text { Correct } \\
\text { detection } \\
\text { rate }(\%)\end{array}$ & $\begin{array}{l}\text { Missed } \\
\text { detection } \\
\text { rate }(\%)\end{array}$ \\
\hline 55 & 1660 & 60.1 & 39.9 & 679 & 64.2 & 35.8 \\
\hline 60 & 2691 & 69.9 & 30.1 & 1232 & 73.6 & 26.4 \\
\hline 70 & 5508 & 84.4 & 15.6 & 2596 & 85.0 & 15.0 \\
\hline 80 & 8640 & 90.0 & 10.0 & 3738 & 91.5 & 8.5 \\
\hline 90 & 11,648 & 91.6 & 8.4 & 4762 & 93.9 & 6.1 \\
\hline
\end{tabular}

All participants were considered in the analysis; however, not all participants experienced hypoglycemic event. 
Table 8. Hyperglycemic Alerts And Detections $(N=262)$

\begin{tabular}{|c|c|c|c|c|c|c|}
\hline \multirow[b]{2}{*}{$\begin{array}{l}\text { Hyperglycemic } \\
\text { alert level } \\
(\mathrm{mg} / \mathrm{dL})\end{array}$} & \multicolumn{3}{|c|}{ Alerts } & \multicolumn{3}{|c|}{ Detections } \\
\hline & $\begin{array}{c}\text { No. of } \\
\text { alerts (n) }\end{array}$ & $\begin{array}{c}\text { True alert } \\
\text { rate }(\%)\end{array}$ & $\begin{array}{c}\text { False alert } \\
\text { rate }(\%)\end{array}$ & $\begin{array}{c}\text { No. of } \\
\text { events (n) }\end{array}$ & $\begin{array}{l}\text { Correct } \\
\text { detection } \\
\text { rate }(\%)\end{array}$ & $\begin{array}{l}\text { Missed } \\
\text { detection } \\
\text { rate }(\%)\end{array}$ \\
\hline 120 & 42,871 & 97.3 & 2.7 & 14,511 & 98.1 & 1.9 \\
\hline 140 & 37,853 & 96.9 & 3.1 & 12,932 & 97.7 & 2.3 \\
\hline 180 & 28,284 & 95.9 & 4.1 & 10,005 & 96.0 & 4.0 \\
\hline 200 & 23,601 & 95.4 & 4.6 & 8586 & 94.3 & 5.7 \\
\hline 220 & 19,091 & 94.6 & 5.4 & 7253 & 92.2 & 7.8 \\
\hline 240 & 14,966 & 93.2 & 6.8 & 5883 & 90.0 & 10.0 \\
\hline 300 & 5193 & 84.3 & 15.7 & 2445 & 76.7 & 23.3 \\
\hline
\end{tabular}

All participants were considered in the analysis; however, not all participants experienced hyperglycemic events.

\section{Discussion}

Studies on the G4 Platinum and G5 Mobile CGM systems have demonstrated an improvement in accuracy across sequential generations of rtCGM systems. ${ }^{10,11}$ Although increased adoption rates of CGM are at least partially attributable to improving accuracy, many patients still face barriers to adoption and continued use of CGM systems such as pain, complexity, the need for frequent sensor changes, toofrequent calibrations, or difficult application. ${ }^{12,13}$ The G6 system's sensor was redesigned for increased stability during its working life and factory calibration.

Factory calibration is particularly desirable as it further diminishes the need for fingersticks and the burden of blood glucose testing. Calibrations may have a beneficial or deleterious effect on CGM accuracy. This is highlighted in Figure 2, which shows with representative in-clinic session data from three distinct participants that calibration can result in improved (Fig. 2A), worsening (Fig. 2B), or no effect on (Fig. 2C) G6 accuracy. In part, this may be related to the accuracy of the meter measurement; meter accuracy is impacted by preanalytical errors, such as user failure to wash hands, ${ }^{14}$ or analytical errors, which occur frequently with some blood glucose monitoring systems. ${ }^{15-17}$ The G6 system maintains the option for users to enter a calibration, which may be useful if CGM readings are discordant with blood glucose measurements.

This study was performed as part of device registration requirements in the United States. The inclusion of $>20,000$ paired YSI-CGM points represents one of the largest data sets used for CGM accuracy analysis to date. The data were collected from adult and pediatric participants whose willingness to undergo supervised intentional glucose manipulations and to wear the sensors for up to 10 days in the outpatient setting allowed for analysis of system performance under a wide range of glucose concentrations, during times of rapid glucose concentration change and across the sensors' working life.

This study demonstrates that the G6 system in the context of a manual applicator has consistent performance in adult and pediatric participants, for all glucose ranges, when glucose is rapidly rising or falling, and across days of wear. Despite extending wear time to 10 days and eliminating the requirement for calibration, the G6 system maintained comparable MARD and \%20/20 with G5 Mobile. The MARD of G5 Mobile was 9\% and 10\% in adults and youth, respectively, whereas the \%20/20 accuracy was 93\% and $91 \%$ in adults and youth, respectively. ${ }^{10,11}$ Consistent G6 accuracy in the hypo- and hyperglycemic ranges manifests in reliable alerts, which may serve to alleviate alarm fatigue and increase user confidence in the system. Moreover, the capacity of the system to accurately alert users to impending hypoglycemia should allow appropriate intervention before the onset of hypoglycemia. Whether this feature results in better clinic outcomes, such as reduction in the number and duration of hypoglycemic events, requires an outcome-based study. Data using the commercialized G6 system, which utilizes an automated sensor applicator, are presented in a companion article and suggest that more consistent sensor deployment mediated by the automated sensor applicator may further improve system accuracy on day 1 and in pediatric subjects. ${ }^{7}$

The benefits of rtCGM use compared with SMBG testing are well established. Numerous studies have established that rtCGM use is associated with reduced A1c, improved glucose

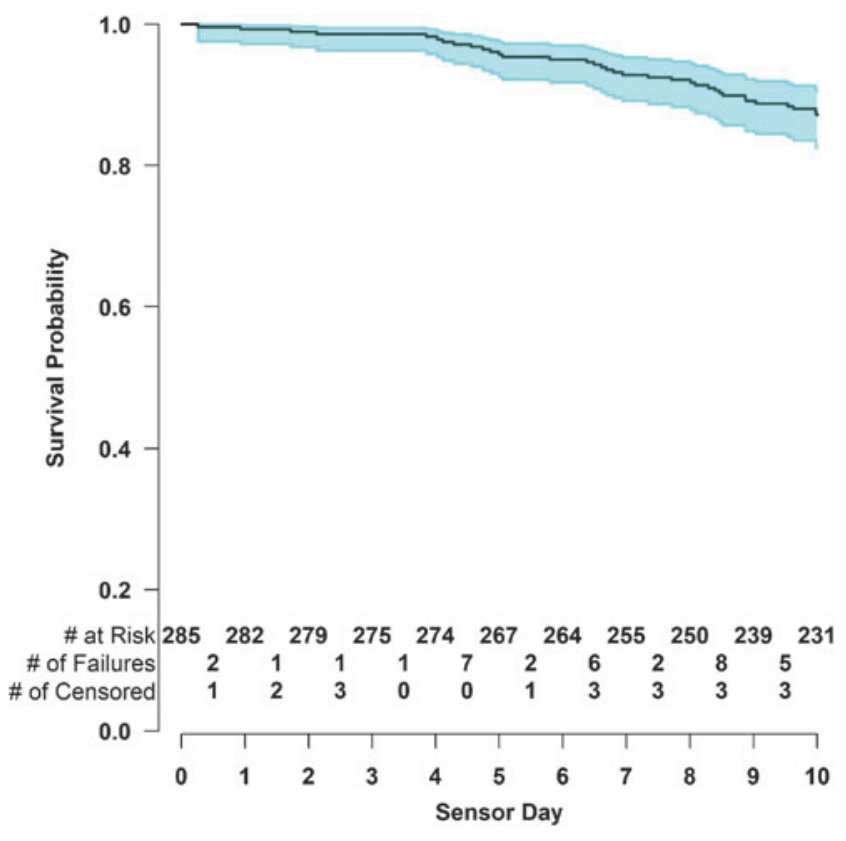

FIG. 1. Kaplan-Meier curve of sensor life $(n=285)$. The shaded area represents the $95 \%$ confidence interval. 

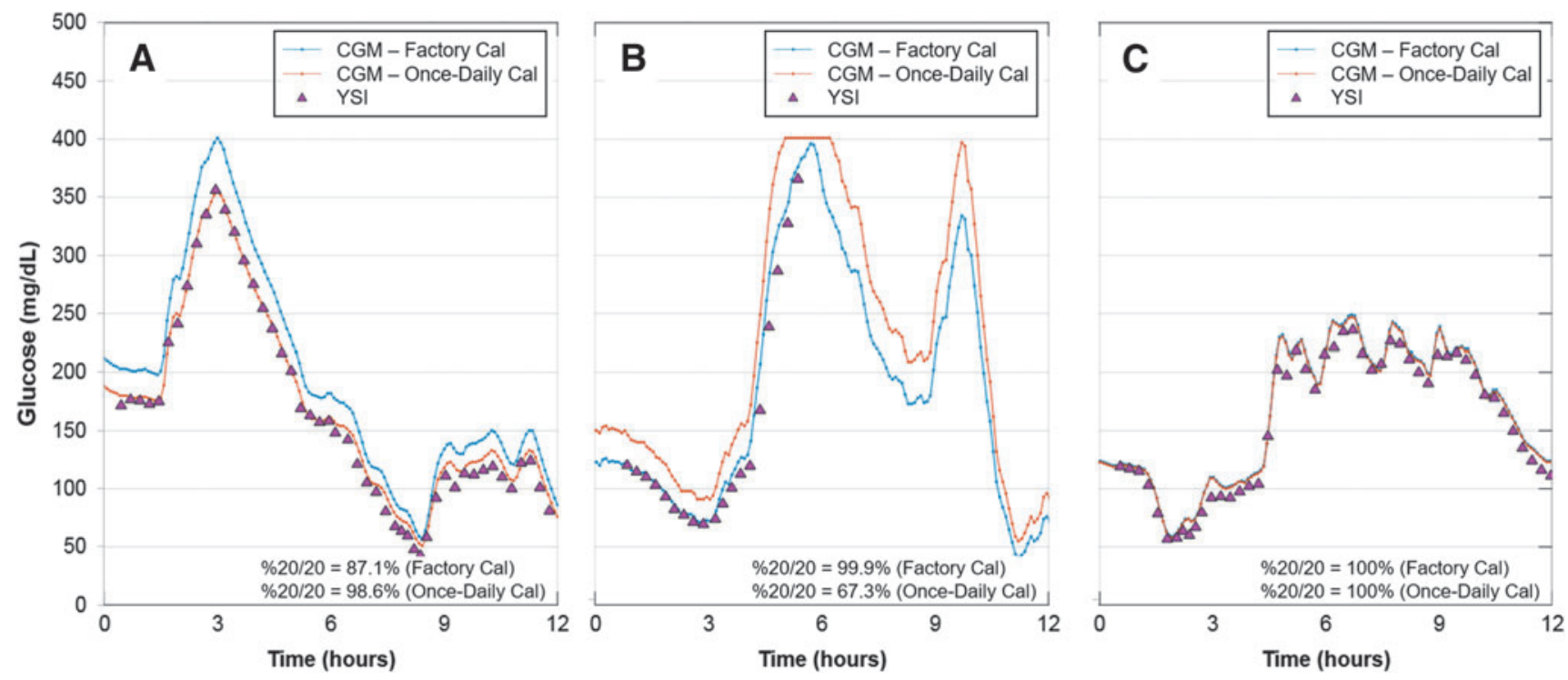

FIG. 2. Comparison of CGM and YSI values with different CGM calibration regimens. Triangles, YSI values; blue lines, CGM values from factory-calibration regimen; orange lines, CGM values from once-daily calibration regimen. (A) Better CGM-YSI agreement with the once-daily calibration regimen. (B) Better CGM-YSI agreement with the factory-calibration regimen. (C) Equivalent performance of the two calibration regimens. CGM, continuous glucose monitoring; YSI, yellow springs instrument.

control, reduced hyperglycemia and hypoglycemia (increased time in range), and improved quality of life compared with SMBG testing. ${ }^{18-24}$ Despite these benefits, a minority of people with $\mathrm{T}_{1} \mathrm{D}^{1}$ and few people with $\mathrm{T} 2 \mathrm{D}$ are currently using CGM. By eliminating the need to calibrate, eliminating acetaminophen interference, simplifying sensor insertion, extending wear time to 10 days, and having consistent performance, the G6 system removes several barriers to using rtCGM, which may lead to increased use of rtCGM in people with diabetes.

\section{Participating Clinical Sites}

The number of randomized participants is noted in parentheses preceded by the site location and site name. Personnel listed are study investigators. Barbara Davis Center for Diabetes, Adult division, Aurora, CO (25): Satish K. Garg; Barbara Davis Center for Diabetes, Pediatric division, Aurora, CO (36): R. Paul Wadwa; William Sansum Diabetes Center, Austin, TX (44): Kristin Castorino; John Muir Physician Network Clinical Research Center, Austin, TX (8): Anna Chang; AMCR Institute, Inc., Escondido, CA (38): Timothy Bailey; Stanford University, Palo Alto, CA (16): Bruce A. Buckingham; Atlanta Diabetes Associates, Atlanta, GA (19): Bruce Bode; Joslin Diabetes Center, Boston, MA (29): Lori Laffel; Rainier Clinical Research Center, Inc., Austin, TX (30): Leslie Joseph Klaff; Diablo Clinical Research Center, Walnut Creek, CA (28): Mark Christiansen; Oregon Health \& Science University, Portland, OR (17): Andrew Ahmann.

\section{Acknowledgments}

This study was supported by a grant from Dexcom, Inc. (San Diego, CA), through the respective universities of the authors. The authors thank the subjects who participated in the study and the research staff at the investigational centers. The authors would also thank Drs. Sarah Puhr, Terri K.
Johnson, and John B. Welsh (Dexcom, Inc.) for their help in preparation of the article.

\section{Author Disclosure Statement}

R.P.W reports research support from Dexcom, Bigfoot Biomedical, MannKind Corporation, Novo Nordisk, Helmsley Charitable Trusty and NIH/NIDDK and advisory board consulting fees from Eli Lilly and Company.

L.M.L. has served as a consultant or on advisory boards for Lilly, NovoNordisk, Sanofi, Roche, Johnson \& Johnson, Boehringer Ingelheim, AstraZeneca, Mannkind, Dexcom, Insulet, Senseonics, Unomedical, and Menarini.

V.N.S.' employer has received research support from the Sanofi US, Dexcom Inc, Eyenuk, and Jaeb Center for Health Research. V.N.S. served on advisory board of Sanofi US and received speaking fees from Dexcom Inc.

S.K.G. has received Advisory Board Consulting fees from Medtronic, Roche, Merck, Lexicon, Novo Nordisk, Sanofi, Mannkind, Senseonics, Zealand, and Eli Lilly. S.K.G. has received research grants through the University of Colorado Denver from Eli Lilly, Novo-Nordisk, Merck, Lexicon, Medtronic, Dario, NCI, T1D Exchange, NIDDK, JDRF, Animas, Dexcom, and Sanofi. S.K.G. does not own stocks in any device or pharmaceutical company.

\section{References}

1. Miller K, Foster N, DeSalvo D, et al.: Continuous glucose monitoring (CGM) use in type 1 diabetes: An update from the T1D exchange clinic registry. Pediatric Diabetes 2016;17(S24):49.

2. Hoss U, Budiman ES: Factory-calibrated continuous glucose sensors: the science behind the technology. Diabetes Technol Ther 2017;19(S2):S44-S50.

3. Olafsdottir AF, Attvall S, Sandgren U, et al.: A clinical trial of the accuracy and treatment experience of the flash glucose monitor freestyle Libre in adults with type 1 diabetes. Diabetes Technol Ther 2017;19:164-172. 
4. FDA authorizes first fully interoperable continuous glucose monitoring system, streamlines review pathway for similar devices. https://www.fda.gov/NewsEvents/Newsroom/Press Announcements/ucm602870.htm Accessed May 14, 2018

5. Clukey R, North B, Parkin C: Utilization of human factors analysis throughout product development enhances usability of CGM sensor applicators. Diabetes 2017;67(S1): A184.

6. Calhoun P, Johnston TK, Hughes, et al.: Resistance to acetaminophen interference in a novel continuous glucose monitoring system. J Diabetes Sci Technol 2018;12:393396.

7. Shah VN, Laffel LM, Wadwa RP, Garg SK.: Performance of a factory-calibrated, real-time continuous glucose monitoring system utilizing an automated sensor applicator. Diabetes Technol Ther 2018;20(6):428-433.

8. Agiostratidou G, Anhalt H, Ball D, et al.: Standardizing clinically meaningful outcome measures beyond $\mathrm{HbA} 1 \mathrm{c}$ for type 1 diabetes: A consensus report of the American Association of Clinical Endocrinologists, the American Association of Diabetes Educators, the American Diabetes Association, the Endocrine Society, JDRF International, The Leona M. and Harry B. Helmsley Charitable Trust, the Pediatric Endocrine Society, and the T1D Exchange. Diabetes Care 2017;40:1622-1630.

9. Danne T, Nimri R, Battelino T, et al.: International consensus on use of continuous glucose monitoring. Diabetes Care 2017;40:1631-1640.

10. Laffel L: Improved accuracy of continuous glucose monitoring systems in pediatric patients with diabetes mellitus: results from two studies. Diabetes Technol Ther 2016;18 Suppl 2:S223-S233.

11. Bailey TS, Chang A, Christiansen M.: Clinical accuracy of a continuous glucose monitoring system with an advanced algorithm. J Diabetes Sci Technol 2015;9:209-214.

12. Engler R, Routh TL, Lucisano JY: Adoption barriers for continuous glucose monitoring and their potential reduction with a fully implanted system: results from patient preference surveys. Clin Diabetes 2018;36:50-58.

13. Shah VN, Boyle C, Garg S, et al.: Human factors associated with insulin pump and continuous glucose monitor discontinuation among adults with type 1 diabetes. Diabetes Technol Ther 2016;18(S1):A18.

14. Hirose T, Mita T, Fujitani Y, et al.: Glucose monitoring after fruit peeling: pseudohyperglycemia when neglecting hand washing before fingertip blood sampling: Wash your hands with tap water before you check blood glucose level. Diabetes Care 2011;34:596-597.

15. Freckmann G, Schmid C, Pleus S, et al.: System accuracy evaluation of systems for point-of-care testing of blood glucose: a comparison of a patient-use system with six professional-use systems. Clin Chem Lab Med 52:1079-1086.
16. Freckmann G, Schmid C, Baumstark A, et al.: System accuracy evaluation of 43 blood glucose monitoring systems for self-monitoring of blood glucose according to DIN EN ISO 15197. J Diabetes Sci Technol 2012;6:1060-1075.

17. Brazg RL, Klaff LJ, Parkin CG: Performance variability of seven commonly used self-monitoring of blood glucose systems: clinical considerations for patients and providers. J Diabetes Sci Technol 2013;7:144-152.

18. Beck RW, Riddlesworth T, Ruedy K, et al.: Effect of continuous glucose monitoring on glycemic control in adults with type 1 diabetes using insulin injections: The DIAMOND randomized clinical trial. JAMA 2017;317:371-378.

19. Lind M, Polonsky W, Hirsch IB, et al.: Continuous glucose monitoring vs conventional therapy for glycemic control in adults with type 1 diabetes treated with multiple daily insulin injections: The GOLD randomized clinical trial. JAMA 2017;317:379-387.

20. Beck RW, Riddlesworth TD, Ruedy K, et al.: Continuous glucose monitoring versus usual care in patients with type 2 diabetes receiving multiple daily insulin injections: A randomized trial. Ann Intern Med 2017;167:365-374.

21. Beck RW, Riddlesworth TD, Ruedy KJ, et al.: Effect of initiating use of an insulin pump in adults with type 1 diabetes using multiple daily insulin injections and continuous glucose monitoring (DIAMOND): a multicentre, randomised controlled trial. Lancet Diabetes Endocrinol 2017;5:700-708.

22. Ruedy K, Parkin C, Riddlesworth T, Graham C.: Continuous glucose monitoring in older adults with type 1 and type 2 diabetes using multiple daily injections of insulin: Results from the DIAMOND trial. J Diabetes Sci Technol 2017;11:1138-1146.

23. Reddy M, Jugnee N, El Laboudi A, et al.: A randomized controlled pilot study of continuous glucose monitoring and flash glucose monitoring in people with Type 1 diabetes and impaired awareness of hypoglycaemia. Diabet Med 2018;35:483-490.

24. Polonsky WH, Hessler D, Ruedy KJ, et al.: The impact of continuous glucose monitoring on markers of quality of life in adults with type 1 diabetes: Further findings from the DIAMOND randomized clinical trial. Diabetes Care 2017; 40:736-741.

Address correspondence to: Satish K. Garg, MD

Professor of Medicine and Pediatrics University of Colorado Denver Barbara Davis Center for Diabetes 1775 Aurora Court, Rm 1324 Aurora, CO 80045

E-mail: satish.garg@ucdenver.edu 